Itinéraires Itinéraires

Littérature, textes, cultures

\title{
Pour une conception non utilitariste du storytelling
}

\section{François Dingremont}

\section{OpenEdition}

\section{Journals}

Édition électronique

URL : http://journals.openedition.org/itineraires/2654

DOI : 10.4000/itineraires.2654

ISSN : 2427-920X

Éditeur

Pléiade

\section{Référence électronique}

François Dingremont, « Pour une conception non utilitariste du storytelling », Itinéraires [En ligne], 2015-1 | 2015, mis en ligne le 18 décembre 2015, consulté le 20 avril 2019. URL : http:// journals.openedition.org/itineraires/2654; DOI : 10.4000/itineraires.2654

Ce document a été généré automatiquement le 20 avril 2019

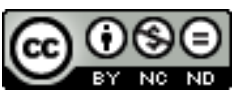

Itinéraires est mis à disposition selon les termes de la licence Creative Commons Attribution - Pas d'Utilisation Commerciale - Pas de Modification 4.0 International. 


\title{
Pour une conception non utilitariste $d u$ storytelling
}

\author{
François Dingremont
}

1 Il existe une infinité de raisons fort justifiées de critiquer les techniques managériales, communicationnelles du storytelling; l'ouvrage de Christian Salmon (2007) en avance un certain nombre. Incontestablement, derrière le récit palpitant et exemplaire du parcours et de la réussite d'un homme parti de rien se trouve une injonction à l'imiter. La construction d'une mythologie autour d'une entreprise créant l'illusion que celle-ci échappe aux lois si terre à terre de l'économie de marché a pour effet de rendre cette dernière plus "fréquentable» que ses concurrentes. Ces récits sont des moyens de séduire un public, de convaincre un consommateur, de souder une équipe, une nation, autour d'une histoire, d'une identité. On prend plus facilement sa part à une culture d'entreprise lorsque son histoire se raconte tel un mythe. Dans ces techniques de communication, le récit enrobe des messages normatifs et injonctifs, provoquant chez celui qui n'y adhère pas la désagréable impression d'être "en dehors du coup ». D'une manière générale, le storytelling est utilisé de nos jours principalement à des fins utilitaristes, voire de manipulation des consommateurs ${ }^{1}$.

2 Parallèlement, Salmon reconnaît volontiers l'importance culturelle, « la vitalité » (2007 : 10), des récits, notamment pour un pays comme les États-Unis. «Les grands récits qui jalonnent l'histoire humaine d'Homère à Tolstoï et de Sophocle à Shakespeare, racontaient des mythes universels et transmettaient les leçons des générations passées, leçons de sagesse, fruit de l'expérience accumulée », ajoute-t-il dans une forme de reconnaissance, malheureusement assez académique, de l'art du récit. Noter l'existence de "grands récits ", de "mythes universels " n'informe pas sur la raison d'être, la persistance et l'efficacité de ces mises en forme narrative de l'existence et de l'expérience que sont les récits de vie et les récits de société. C'est sur ce versant que nous allons porter notre analyse.

3 L'ouvrage de Salmon est, en effet, une excellente critique du storytelling à visée manipulatoire. Pour autant, on aurait pu la concevoir et peut-être lui accorder une plus 
large pertinence si elle tenait compte de ce qui la distingue moins de l'univers des grands récits ou de la pensée conceptuelle, critique, émancipatrice, que de la manière où elle n'apparaît jamais à la hauteur des ambitions et des possibilités qui fondent l'art du récit ${ }^{2}$. Pour le dire plus clairement, si le storytelling fait écho et joue sur le plaisir de dire et d'écouter des histoires, élément souvent négligé dans l'approche critique, son utilitarisme le dessert.

4 L'utilisation instrumentale des récits de société ou de vie met néanmoins en lumière une base anthropologique essentielle à analyser pour fonder une approche critique respectueuse de la complexité de la sensibilité humaine. En effet, l'homme est naturellement attaché non pas aux récits en général, mais à ceux qui se distinguent par leur qualité, leur originalité et leur potentialité.

5 Si la différence entre "grand récit » et procédé du storytelling est avant tout qualitative, mais aussi éthique, il n'en demeure pas moins que l'un et l'autre possèdent, à des degrés divers, une réelle efficacité. Ils touchent, en effet, à un élément essentiel de la construction symbolique des individus, de notre ontologie, à savoir l'attachement à une pensée qui n'est pas exclusivement conceptuelle mais aussi narrative. C'est sur cet aspect que nous attirerons les attentions, sur l'importance, si l'on se préoccupe d'anthropologie générale, de considérer en complément du sujet pensant sur lequel la tradition philosophique s'est construite, un homo narrans et un homo fabulator ${ }^{3}$.

Nous n'aurons pas comme objectif de construire un discours scientifique et critique sur la place des récits de vie dans notre quotidien, sur la manière dont on peut les considérer et les étudier à la lumière de données sociologiques ou d'un savoir narratologique sensible aux contextes et procédures d'énonciation, mais de pointer ce qu'ils disent et apportent sur l'homme et sa condition. Pour nous mettre sur la voie d'une compréhension de l'efficacité non utilitariste des récits, nous nous appuierons essentiellement sur les travaux de Jerome Bruner.

Notre principal outil pour mettre de l'ordre dans l'expérience, pour forger une sorte de continuité entre le présent, le passé et le possible est le récit, la narration [...]. Le récit est notre moyen spécifiquement humain de mettre en ordre les événements au fil du temps, de les ordonner de manière distincte qui prenne en compte les états et les circonstances dans lesquels se trouvent les protagonistes, et qui en même temps laisse une marge de manœuvre à celui qui raconte l'histoire et à ceux qui l'écoutent. C'est par excellence l'outil de construction de la culture. (Bruner $2000: 8)$

7 Le problème consiste à mesurer si ce qui est dit ici sur le récit est recevable pour le storytelling. En d'autres termes avons-nous, affaire à deux réalités narratives totalement hétérogènes ou à deux modalités d'une même fonction, d'une même pratique, à savoir celle de raconter des histoires? Un détour par l'Antiquité nous aidera à positionner les termes du débat. En effet, si l'on se penche sur la longue tradition occidentale des récits on s'aperçoit qu'Homère, dans l'Odyssée, pose les bases d'une pratique bien particulière de la narration. Ulysse, composant devant les Phéaciens un récit de vie, apparaît bien comme le prototype du storyteller. Nous verrons donc en premier lieu ce qui ancre sa pratique discursive dans cet univers. D'ailleurs, la réception de la tradition homérique, avec les débats qu'elle a suscités dans l'Antiquité, est le lieu où se distinguent les prémisses d'une dichotomie entre pensée narrative et pensée conceptuelle, discussion que nous aborderons dans un second temps. Le recours aux récits de vie fut très tôt jugé, dans les cercles philosophiques ou philologiques ${ }^{4}$, comme superflu, vain, infantile, voire trompeur. Ensuite, nous passerons en revue un certain nombre d'arguments utiles pour se défaire 
du présupposé de la supériorité du concept sur le récit ${ }^{5}$. En ayant soin de ne pas confondre contes et storytelling, nous nous attacherons néanmoins à mettre en lumière que, dans les deux cas, le contexte est toujours celui que Joseph Russo (1976) avait appelé « contexte d'auralité ». Nous verrons ensuite, en nous arrêtant sur deux récits de vie qui ont marqué notre dernière décennie (Joe le Plombier et Oscar Pistorius), que ce qui rend finalement plus marquant leur histoire, c'est moins le message qu'elle véhicule que la manière par laquelle une fois prise dans le jeu de l'auralité et des péripéties, la « machine à formater » se grippe. Le renversement des fortunes des héros promu par les stratèges du storytelling rappelle que l'univers des récits n'est pas celui assertif de la vérité, mais subjonctif, terme cher à Bruner, des possibles.

\section{Storytelling dans l'Odyssée}

8 Dans Les Deux Sources de la religion et de la morale, Bergson considère que la fonction fabulatrice propre à l'homme fait contrepoids «au pouvoir dissolvant de son intelligence» (1932: 137) ${ }^{6}$. La tradition philosophique platonicienne, cartésienne ou pascalienne projetait, à l'inverse, l'idée que la pensée et la raison étaient les meilleurs moyens de dissoudre le voile et les brumes des illusions de l'imagination. Cette puissance de dissolution était l'un des principaux attraits de la philosophie. Imagination et raison n'étaient pas mises au même niveau. Bergson voit juste lorsqu'il invite à considérer conjointement et non pas séparément effort de l'intelligence et possibilités de l'imagination. Pour autant, il épouse une opinion qui fait débat. Si philosopher, c'est apprendre la clairvoyance, la première consiste en la capacité de distinguer ce qui essentiel de ce qui ne l'est pas, nous apprend-on dans les manuels scolaires d'introduction à la philosophie. Pour ce faire, si l'on suit Platon, seul le raisonnement dialectique compte, car, d'après lui, il ne se laisse pas charmer par le chatoiement trompeur des récits fabuleux, ce en quoi il vise Homère ${ }^{7}$. À l'aspect dispendieux des récits traditionnels, la philosophie platonicienne et aristotélicienne, faisant la chasse aux paradoxes, aux équivoques, aux opacités, bref au surplus non maîtrisé de notre fonction fabulatrice répond par une parole, un raisonnement et une pensée économes. Ainsi, la figure du philosophe d'inspiration socratique ou cynique est à mille lieues de cet incorrigible bavard qu'est Ulysse. Homère et Ulysse sont des fabulateurs. Le sont-ils au même titre et selon la même logique? Les recherches sur la poétique de l'odyssée ont orienté la réflexion vers une nette distinction entre deux instances gouvernant la parole ondoyante, chatoyante dans ce texte, d'un côté le poète/aède, tels que l'incarnent Démodokos et Phémios, de l'autre le storyteller qu'est Ulysse (Mackie 1997, Scodel 1998, Beck 2005). Dans les deux cas, il s'agit bien de récits faits devant un auditoire et d'un effet: un silence admiratif décrit comme le passage d'un charme (terpsis ou klèsis) ${ }^{8}$. Les aèdes sont inspirés tandis qu'Ulysse a à sa disposition non pas la vision des Muses, mais une imagination tournée vers la composition d'un récit de vie. Loin d'envisager de chanter la gloire des héros du passé, l'Odyssée débute avec une invocation aux Muses où l'aède demande l'inspiration pour narrer non pas le temps d'un passé lointain et glorieux mais pour dire ( ennepein) « l'Homme aux milles tours ${ }^{9}$ ». La focalisation sur le récit de vie ne disparaittra pas. Télémaque quittera Ithaque à la recherche de nouvelles, de rumeurs, de bruits concernant son père. À côté du kléos, de la gloire que les aèdes célèbrent, s'installe avec les récits d'Ulysse, l'ainos, que les hommes fabriquent s'appuyant sur des histoires, souvent des récits de vie véhiculés de bouche à oreille. C'est cette matière que l'odyssée et 
Ulysse travaillent. Devant les Phéaciens, Ulysse performe le récit de son errance ${ }^{10}$. Le héros est donc logiquement qualifié de poluainos ${ }^{11}$. Pietro Pucci (1996:192) écrit que l'adjectif composé poluainos est « l'épithète fixe, exclusif pour Ulysse qui le caractérise comme celui qui utilise les récits pour survivre et pour s'assurer un succès et une récompense auprès de ceux qu'il croise et auxquels il s'adresse ». Gregory Nagy (1994:281) note que l'ainos « désigne un mode de discours poétique approprié à un dessein qui va bien au-delà de la simple louange [...] il est un conte allusif, contenant un dessein caché ». Ulysse fait son apologie en utilisant une technè narrative ${ }^{12}$. La technè est un savoir et un savoir-faire qui relève de l'adresse, de l'habileté, de l'industrie. Les termes employés ici montrent que nous ne sommes pas éloignés de l'univers de la manipulation, voire de la tromperie. La performance d'Ulysse dure toute la nuit, sans que l'attention de son auditoire ne baisse. Il joue sur les affects, les émotions que sa narration suscite. La longueur du récit vient de l'importance de ce qu'il espère obtenir en retour. Il cherche la reconnaissance de ses hôtes, afin qu'ils le ramènent à Ithaque, si possible accompagné d'un butin. Devant Eumèe, au chant XIV (462-508), Ulysse fait un si beau récit, que ce dernier s'empressera de rapporter son expérience d'auditeur à Pénélope ${ }^{13}$. Les ainoi ulysséens font la joie d'Eumée, ils attirent ses faveurs ${ }^{14}$. En effet, suite à la belle narration que le héros fait d'une scène de l'expédition à Troie, Eumée lui donnera le manteau qu'il convoitait. Tous ces éléments constituent une sorte de socle sur lequel repose une forme traditionnelle de storytelling que les Anciens prenaient soin de différencier de la manière aèdique de produire un récit. Point d'inspiration, de vision chez Ulysse, juste le talent de faire coïncider un récit avec une situation présente ${ }^{15}$. Le héros ne cesse de parler de lui-même, toutes les histoires qu'il raconte tournent autour de sa personne. Ce schéma intéressé, centré sur soi, nourri par l'expérience de la vie, est terriblement efficace quant au gain qu'il obtient en jouant sur la sensibilité de son auditoire. Cette efficacité est très éloignée de celle du chant de l'aède dont l'objectif unique est de créer du plaisir en glorifiant les figures héroïques du passé. La proximité et la distinction entre storytelling, récits particuliers où sont scénarisés des récits de vie et poésie divinement inspirée, portant sur un passé mythique sont déjà fortement marquées dans l'odyssée. Pour Platon et plus tard les stoïciens, nous avons affaire, dans les deux cas, à des inventions inutiles, peu dignes des esprits formés à la philosophie.

Intérêt, volonté de séduction, jeu sur les émotions, les récits d'Ulysse sont des exemples réussis de storytelling. Ils sont réussis car précisément, ils ne sont pas le fruit d'un programme narratif conçu à l'avance, tels qu'on les imagine façonnés dans les laboratoires des agences de communication, mais d'une invention permanente et d'une imagination jamais en sommeil. Si dans les deux cas, on peut parler d'une technè narrative, le sens du terme technè n'est pas le même et probablement toute la différence est là. La technè d'Ulysse est celle que l'on retrouve dans beaucoup de récits traditionnels, elle correspond plus ou moins à ce que les anthropologues mettent sous l'étiquette des pratiques des trickster ${ }^{16}$. Inventivité, habileté en sont les ressorts. L'inattendu, l'univers fantastique, en un mot, le charme qui est déployé dans les récits ulysséens est aux antipodes de la séduction racoleuse de la technè du management, assez peu inventive et rarement habile. En revanche, nous avons vu que la question de l'intérêt, du gain, de la récompense, loin d'être conçu ou de nécessiter forcément un formatage des esprits peut être un jeu où les deux parties, locuteur et récepteur, sont gagnantes. Le storytelling, lorsqu'il est utilisé avec talent, peut s'avérer être un intermédiaire entre poésie désintéressée et techniques de séduction. 
Le storytelling, qu'il soit traditionnel ou contemporain, met en perspective les interactions entre un « moi » sujet d'expérience, un « je » instance narrative de ces expériences, et un « nous » qu'il s'agit de construire et de souder autour du récit ainsi produit. Les mises en scène, les scénarisations qu'il suscite varient du plus élémentaire au plus subtile.

\section{Statut non philosophique des récits?}

11 Le philosophe stoïcien Sénèque avoue qu'il a les plus grandes difficultés à comprendre pourquoi Chrysippe (philosophe stoïcien $\mathrm{du} \mathrm{III}^{\mathrm{e}}$ siècle avant notre ère), pourtant «si remarquable par cette finesse d'esprit qui pénètre au fond des choses, et va droit au but, sans perdre plus de paroles qu'il n'en faut pour se faire comprendre», se perdait nonobstant en "vaine érudition ", en portant intérêt aux représentations homériques, toutes relevant, selon lui, de la plus commune « niaiserie ${ }^{17}$ ». La tradition philosophique, en se détournant de la pensée narrative, fabrique un système hiérarchique en promouvant le sujet pensant au détriment de l'homo narrans et fabulator. Une des particularités de la pensée occidentale est d'ailleurs de concevoir comme nettement séparées pensée conceptuelle, économe, maitrisée, " pénétrant au fond des choses » et récit, dispendieux, non maîtrisé en temps et paroles, bref superficiels. D'une certaine manière, le principe d'une économie de la parole doit beaucoup à la confrontation entre sophistes et philosophes de la Grèce classique. Dans la critique, souvent caricaturale, que font les seconds des premiers, on retrouve une méfiance vis-à-vis des usages non conceptuels des mots. Le « plaisir de parler » des sophistes, comme le remarque Barbara Cassin (1986: IX), est jugé vain une fois que la philosophie aristotélicienne pose comme vertu supérieure de la pensée de se concentrer sur le principe de non-contradiction et le régime de signification. Les récits de la tradition orale ne sont pas pensés avec la finalité d'un éclairage du sens et ne sont pas astreints de respecter le principe de noncontradiction. D'après François Flahault, le conte de tradition orale ne donne pas son propre modèle explicatif, c'est la tâche de celui à qui il s'adresse, il ajoute, "pour l'auditeur ou le lecteur, ce n'est pas le message du conte qui importe d'abord - à supposer qu'il y en ait un - c'est l'effet que le récit produit sur lui : il vit comme des fins en soi l'étoffe du récit, sa durée, et les impressions qu'il éprouve. C'est à travers ce plaisir vécu que le récit contribue à mettre en sens son expérience» (Flahault 2001: 7). Flahault ajoute :

Interpréter un récit de fiction ne consiste pas à le « décoder » comme on traduirait un message d'une langue dans une autre en laissant de côté les émotions éprouvées. Celles-ci, au contraire, font partie du récit, elles en sont la chair. Pour analyser un récit, il faut essayer de mettre des mots sur les impressions qu'il suscite en nous; et remonter, en s'appuyant sur l'étude de sa structure, aux causes du plaisir qu'il procure. (Ibid. : 10)

Émotion, plaisir, pathos sont aussi les bases du storytelling actuel, une critique de cette pratique s'appauvrit en ne leur accordant pas une place qui dès Homère s'imposait. De plus, à être trop catégoriquement critique vis-à-vis des recours à la narrativité on court le risque de perpétuer un présupposé qui a longtemps couru dans la sphère de la réflexion philosophique, à savoir que le concept, dans sa toute sa puissance, déploie son intelligibilité sans avoir recours à des supports narratifs. Tel était l'avis par exemple des néoplatoniciens. Maxime de Tyr considérait les modèles narratifs de la tradition homérique intéressants pour celui qui envisage la vie sous l'angle de l'action, mais, pour lui, il est de meilleur aloi de se tourner vers la contemplation. Cette dernière n'a guère 
besoin de mythes, de récits pour nous faire toucher le beau ${ }^{18}$. La conceptualisation philosophique peut être entendue comme un mouvement vers l'abstraction. En ce sens les récits, parce que dépendants d'instances narratives et donc assujettis au hic et nunc de l'énonciation, ne nous permettent pas de parcourir le chemin vers un monde des idées délesté de toute pesanteur spatio-temporelle. Ainsi pour saisir, par exemple, ce qu'est la vertu, son essence, le philosophe apprendra à se débarrasser de modèles véhiculés par les traditions poétiques, afin de penser la vertu par et pour elle-même. Le travail spéculatif de la philosophie est une voie de détachement de l'inconsistance des images, des histoires, en un mot de la narrativité. Les sensations, les affects seraient censés troubler le travail de l'intellection, de l'entendement.

Tout en apportant de précieux moyens de penser l'homme tel qu'il est, la connaissance rationnelle tend aussi à voir l'homme telle qu'elle est: le sujet de la connaissance rationnelle tend à projeter son reflet dans son objet, à se représenter l'homme à son image. Et ce n'est certainement pas en qualifiant d' "affectif ", d'« émotionnel » ou d' « irrationnel » le versant de l'être humain qui lui échappe que le sujet connaissant combat efficacement sa propre méconnaissance. (Flahault $2001: 14)$

La pensée logico-scientifique, selon Jerome Bruner, «se préoccupe des causes générales, cherche à les établir» (2000: 29). Elle est guidée "par des hypothèses régies par des principes » (Ibid.). La pensée narrative s'appuie sur l'imagination:

[...] ce mode de pensée s'intéresse à l'intention et à l'action humaines (ou à l'image de l'homme), aux vicissitudes et aux conséquences qui affectent leur accomplissement [...]. Le mode paradigmatique cherche au contraire à transcender le particulier en allant toujours plus loin dans l'abstraction, au point d'aboutir à nier par principe toute valeur explicative à ce qui procède du particulier. (Ibid. : 30)

Pourtant, comme l'écrit François Flahault (2001: 11), « la pensée conceptuelle pousse sur un terreau de sentiments, d'émotions, d'images plus ou moins interconnectées, de scènes plus ou moins organisées en récits, de manières d'être ou de postures inconscientes ». L'auteur poursuit en invitant :

À étudier des récits de fiction qui circulent dans notre culture en les regardant comme des mises en forme de l'expérience humaine. Ces récits ne relèvent pas de la pensée philosophique (ils n'ont pas cette dignité), mais, précisément en tant qu'impensé, ils appartiennent à ce terreau sur lequel toute pensée, même philosophique, se développe. Rien en effet ne permet d'affirmer a priori que la mise en forme de l'être-au-monde qui sert de socle à la philosophie reflète plus fidèlement la réalité humaine que la mise en forme de l'expérience dont témoignent la tragédie grecque, la littérature, les mythes africains, les contes, ou, tout simplement, la manière dont nous nous orientons pratiquement dans la vie quotidienne. (Flahault $2001: 11$ )

Derrière l'analyse critique que l'on peut adresser aux pratiques de storytelling la question de la validité théorique et heuristique du particulier et du singulier se pose. Comment ne pas en passer par le particulier ou le singulier pour aborder d'une manière juste des thématiques fondamentales telles que la souffrance ou le désir? Ce sont évidemment sur ces paramètres que joue le storytelling politique ou médiatique en les exacerbant. Les émotions, les passions sont les matières des récits car elles sont celles de l'existence et de l'expérience. Avec Yves Citton (2010:12), nous pouvons ajouter à ce qui vient d'être présenté que « la structuration narrative constitue une précondition nécessaire à l'action humaine, en même temps qu'un horizon appelé à opérer l'intégration de nos divers gestes quotidiens $»$. 
16 Accent mis sur l'ordinaire, le quotidien, le storytelling met en lumière les interconnexions entre les imaginaires individuels et collectifs.

\section{Auralité}

17 Les critères d'appréciation des deux pensées auxquelles fait allusion Bruner ne se recoupent pas : « on estime qu'une histoire est "bonne" (qu'elle soit présentée pour vraie ou comme pure fiction) en faisant appel à des critères d'une autre nature que ceux qu'on utilisera pour juger qu'une argumentation est juste» (Bruner $2000: 28$ ). Le jugement sur les récits de vie ou de société met en relief une certaine caractéristique des sociétés anciennes et contemporaines, à savoir leur dimension d'auralité. Le storytelling est une technique qui ne crée pas son matériau mais assemble, accommode, des bouts de narrations entendus à droite à gauche, de bouche à oreille, avec comme objectif de créer de l'effet sur un destinataire. Le terme d'auralité rend compte de l'importance de ce dernier. Le storytelling d'Ulysse fonctionne car la société homérique est "aurale», auditive, pour reprendre le terme anglais de Joseph Russo. Employer le terme « aural» permet de mettre l'accent sur la circulation de la parole dans les sociétés où les liens entre écoute, imitation et mémorisation se font sans le recours à l'écrit.

Le chemin qui va d'interprète en interprète, quand il n'est pas frayé par l'écrit, ne peut s'ouvrir qu'avec l'assentiment de la communauté et grâce à la complicité du groupe dont la mémoire se parle [...]. Pour entrer et prendre place dans la tradition aurale, un récit, une histoire, une œuvre de parole quelle qu'elle soit, doit être entendue, c'est-à-dire acceptée par la communauté ou l'auditoire à qui elle est destinée. (Detienne 1981 : 84)

Florence Goyet (2006: 561) choisit aussi d'employer ce terme pour rappeler que les épopées « sont des textes auraux, façonnés dans et par la présence du public ». C'est bien cette présence et ces exigences qui sont au centre du storytelling. Présence du récepteur qu'il s'agit de séduire, de convaincre et de manipuler comme Ulysse le faisait avec Eumée, le porcher d'Ithaque. Le héros recherche l'assentiment de son interlocuteur.

L'ondoiement des récits dans l'univers de l'auralité rend difficile leur validation selon les critères du vrai ou du faux ou d'une connaissance scientifique. Leur référent n'est ni l' epistémè ni la vérité, mais la doxa :

La doxa unit ce qui s'est écartelé entre un sens subjectif : ce à quoi l'on s'attend, ce qu'on croit, ce qu'on estime bon et un sens objectif : ce qui apparaît, ce qui paraît, ce qui semble. La courbe du sens possède à chaque fois une amplitude maximale de valeur qui va du plus négatif au plus positif: de l'hallucination («opinion fausse, imagination, conjecture ») à la justesse normative de l'idée admise ("attente, estime, conjecture, croyance, dogme, réputation»), et de l'apparence trompeuse («illusion, faux-semblant») à l'apparition dans toute sa splendeur. (Cassin 2004 :

Le vaste spectre sémantique de la doxa, du plus négatif au plus positif, correspond dans son étendue aux différentes gammes de storytelling. Les doxai sont les savoirs, les traditions, les conventions sociales, les usages, ce qui nous parle. Cette dernière formule, laissant entendre là aussi le meilleur comme le pire, trouve sa place au cœur des stratégies du storytelling. Pour autant, on aurait tort de perdre de vue l'ambivalence de l'univers doxique. «La doxa, loin d'obscurcir nos jugements et notre vision du monde, nous offre un moyen de comprendre la complexité des interactions ", écrit la philosophe Anne Cauquelin (1999: 18). Étudier les récits, avec cette toile de fond de l'auralité et de la 
doxa est une manière d'avoir prise sur les phénomènes interactionnels. Insaisissable, ondoyante, la doxa, vue par les philosophes sans l'ambivalence du pire et du meilleur qui la constitue, est l'outil de ceux, habiles, qui en font un instrument de manipulation et de contrôle. Plus grave, comme mode de gouvernement, elle rabaisse la politique à la gestion des opinions. Puisque l'art de raconter des histoires, ou plus simplement le plaisir de parler, est vu dans les premiers temps de la philosophie comme une pratique doxique de sophistes, on comprend l'héritage qui pèse sur la pratique du storytelling.

21 La question du type de paroles qui prend en charge les récits de société est donc philosophiquement sensible. Fait-elle de celui qui reçoit ces discours un auditeur passif, voire manipulé, formaté ou au contraire un individu actif, participant? C'est certainement dans une position médiane que se situe la réponse la plus juste à cette question. Croire que le storytelling est une "machine à formater les esprits » est une opinion qui tend à surestimer, afin de mieux la critiquer, l'aspect maîtrisé de cette pratique.

\section{Les péripéties}

Le storytelling du marketing s'illusionne quant à sa capacité à maitriser totalement les récits qu'il construit. Nous allons voir à l'aide de deux exemples que ce n'est qu'à travers des péripéties non anticipées, non pensées par les conseillers en communication que deux moments forts du storytelling médiatique, au départ parfaitement formatés et donc très faibles d'un point de vue narratif, sont devenus deux très bons récits. "Une histoire commence lorsqu'apparaît une sorte de brèche dans l'ordre des choses auquel nous nous attendons ", écrit Jerome Bruner (2002: 19). Il faut pour cela le passage par l'étape des péripéties. La péripétie est l'instant, toujours selon Bruner, où «quelque chose va de travers » (Ibid. : 28). Les exemples de Joe the Plumber et d'Oscar Pistorius montrent que la dramaturgie de certains récits de vie peut échapper totalement au carcan de la communication institutionnelle, par le fait même que les sujets et les objets traités ne sont pas des créations littéraires ou philosophiques, mais des réalités vivantes, donc ondoyantes et changeantes.

Lors de la campagne présidentielle aux États-Unis opposant John McCain à Barack Obama, ce dernier, lors d'un déplacement, est pris à partie par un certain Joe Wurzelbacher. L'homme se présente non seulement comme un plombier, mais aussi comme incarnant le "rêve américain ", récit de société par excellence ${ }^{19}$. Les spécialistes ont choisi de faire de Joe l'incarnation de la rencontre entre récit de vie et récit de société. Avant de rentrer dans le cœur du récit, il est bon de rappeler ce qu'est le rêve américain. Le "Yes we can! » de Barack Obama entre dans une tradition que l'on pourrait résumer en disant que les États-Unis, à la différence de n'importe quel autre pays, offrent la possibilité de réussir à quiconque faisant preuve de courage, de détermination et d'esprit d'entreprise. Ce rêve est donc constitué d'une infinité de micro récits de vie ayant suivi cette trame, le parcours d'Obama en fait par exemple partie ${ }^{20}$.

24 Joe interpelle donc le candidat Obama en affirmant que s'il est élu, il n'aura plus les moyens financiers de maintenir à flot une entreprise qu'il a pourtant créée de ses propres mains, travaillant « dix à douze heures " par jour. Travailler durement, investir, créer sa propre entreprise, bref être un self-made-man, tel est un des storytelling qui épouse le mieux les contours de l'American Dream. Face à Joe, Obama passe pour le briseur d'un rêve 
que le plombier dit si bien incarner. Ce récit fera mouche, l'anecdote sera citée vingt-cinq fois lors du dernier débat télévisé opposant Obama et McCain. Laissons la chute pour plus tard et prenons un autre exemple avec le cas de l'athlète handicapé Oscar Pistorius. Ce dernier, bien qu'amputé des deux jambes et muni de prothèses, est le premier coureur handicapé à participer à une course internationale avec des valides. Après sa participation aux mondiaux d'athlétisme à Daegu dans l'équipe sud-africaine du $4 \times 400 \mathrm{~m}$, puis aux Jeux olympiques de Londres de 2012, il est surnommé "The blade runner » et s'attribue lui-même le titre de " chose la plus rapide du monde sans jambes » (voir Marcellini 2010). Comme dans le cas précédent, la trame narrative joue sur une dichotomie simple et à forte charge émotionnelle, où l'effort, la persévérance sont en proie à l'incompréhension, voire l'injustice des institutions. En effet, il a fallu que Pistorius et ses nombreux avocats bataillent fermement pour obtenir son acceptation parmi les coureurs valides. S'il parait en effet injuste de faire payer à Joe le plombier des impôts d'un montant qui risque de mettre à mal l'entreprise qu'il a bâtie à la force de sa volonté et de son courage, il paraît aussi a priori injuste d'empêcher Pistorius de participer à d'autres épreuves avec des valides, lui aussi étant un exemple de persévérance et de réussite individuelle. Dans les deux cas, l'État imposant ses règles de fonctionnement (taxation et séparation entre valide et non-valide) apparaît comme une instance aveugle et sourde à la réussite individuelle et aux progrès de la science, aux promesses d'émancipation qu'elle met en perspective. Pistorius incarne de surcroît le dépassement de la finitude de la condition humaine, le surhomme. Le scénario est très efficace, la trame narrative du self-made-man face à l'injustice sociale, de l'homme en symbiose avec la technologie est parfaitement rodée, les storyteller ne cessent de l'exploiter. Les émotions étant mobilisées par une dichotomie des plus simplistes, par un récit de vie qui ne saurait laisser indifférent, les messages passent d'autant mieux, en l'occurrence les limites des politiques fiscales et des réticences présentées comme d'un autre âge face au prométhéisme scientifique offrant à l'homme les possibilités de dépasser les limites de sa nature.

Le cadre de réflexion que nous venons d'évoquer ne correspond qu'à l'aspect le plus saillant des techniques de communication du storytelling. Elles supposent une sorte d'aplanissement des éléments les plus opaques de l'auralité. En effet, puisqu'il s'agit de récits qui apparaissent exemplaires dans une optique managériale, ironie, décalage, renversement, bref tout ce qui peut ternir la positivité béate et naïve de l'idéologie managériale est proscrit ${ }^{21}$. Le piège de l'auralité s'est néanmoins refermé sur les storyteller . S'emparant du récit de Joe, le montant en épingle, ils ont cru le maîtriser. Grossière erreur. En effet, après la campagne électorale américaine, la presse rapportera que Joe le plombier s'appelle Samuel, n'a jamais créé d'entreprise, mais était employé de la société Newell Plumbing - le mythe de l'auto-entrepreneur en prenait un coup - et de surcroit, il était en délicatesse avec les services du fisc américain. Il se présentera, par la suite, aux primaires républicaines dans l'Ohio ${ }^{22}$. Le récit concernant Pistorius fournit lui aussi un renversement à l'ironie encore plus grinçante. Le 14 février 2013 (jour de la SaintValentin), il écorne sa réputation de héros national ; en effet il abat d'un coup de pistolet sa compagne. Si l'on en restait là, l'histoire serait un simple fait divers, mais un détail la fait basculer du côté de la pragmatique narrative que l'on retrouve dans les contes de tradition orale, à savoir le renversement des sorts et des fortunes par la transformation du statut du « détail » qui offrait au héros, à un moment donné du récit, la possibilité de sa victoire ${ }^{23}$. Dans les deux cas que nous étudions, les fortunes sont renversées dans un sens inattendu. Oscar Pistorius, pour sa défense, plaide la panique, il dit avoir tiré 
précipitamment, de son lit, pensant que des cambrioleurs s'étaient introduits dans son domicile. Pour démonter l'argument de la précipitation et de la panique, l'accusation cherche à déterminer si lorsqu'il a fait feu, il portait ses prothèses. Si tel était le cas cela supposerait un délai avant le coup de feu et donc si ce n'est une forme de préméditation, tout au moins un enchaînement événementiel qui échappe à la panique. Les prothèses, éléments clés de la success story de Pistorius deviennent dans cette hypothèse, des éléments à charge : le carrosse devient citrouille. L'outil de sa réussite, ce don qui nous fait penser aux rencontres prodigieuses des contes, à l'acquisition de formules ou de choses au pouvoir magique, pourrait s'avérer être également celui de sa chute. Ce fait n'est pas sans rappeler ce que Flahault observe à propos des contes de la tradition orale et la « logique du renversement du complément trop désiré en objet en-trop» (2001: 50). L'élément le plus marquant du storytelling se retourne contre le héros.

Il y a là une sorte d'ouverture ironique du récit que prennent rarement en compte leurs concepteurs et ceux qui font la critique du stotytelling. Mettant en relief les renversements, les erreurs, la non-maîtrise, ces récits de «ce qui va de travers " (Bruner 2002: 30) sont des piqûres de rappel concernant la condition humaine. Leur intérêt narratif est bien supérieur à ceux que construisent a priori les spécialistes en communication. On ne saurait trop leur conseiller de méditer cette observation de Bruner : «ce qui permet à l'histoire de réaliser ces prodiges, ce n'est pas seulement sa structure en soi, c'est aussi sa flexibilité, sa malléabilité » (Ibid. : 31). L'efficacité narrative de ces deux récits se situe moins dans le fait que nous avons affaire à des mensonges, ce qui conviendrait pour une critique morale, mais dans les détails et les péripéties non maîtrisables qui font passer du statut de héros à celui de réprouvé. Le jugement porté sur ces deux récits ne se réclame pas d'une extériorité critique fondée sur la révélation des manipulations, d'un raisonnement argumenté, mais d'une attention au cours non maitrisable du déroulement d'une vie, d'une existence.

\section{Une approche non utilitariste des récits}

En tenant compte des aspects évoqués précédemment, nous sommes en mesure de nuancer les craintes exprimées par Christian Salmon. Si l'émotionnel triomphe bien dans le storytelling, s'il se substitue souvent aux arguments raisonnés, il semble bien difficile de le maîtriser aussi parfaitement et complètement que ne le suggère l'auteur, le diable étant dans les détails. Par ailleurs, nous avons aussi tenté de montrer que les récits de société ont une propension, précisément parce que leurs effets ne sont pas toujours contrôlables et que les histoires ne s'arrêtent pas toujours là où les professionnels de la communication le souhaiteraient, à subvertir involontairement le message qu'ils étaient censés diffuser. Enfin, il est évident que lorsque l'accent est à ce point mis sur des récits de vie, sur des fortunes, le principe du renversement s'introduit souvent insidieusement. Une existence se déroule rarement d'une façon purement linéaire.

Le don de raconter des histoires caractérise l'homme autant que la station debout ou l'opposition du pouce à l'index. Tout indique que c'est notre manière « naturelle » d'utiliser le langage, dans le but de caractériser les déviations qui, sans cesse, viennent perturber le cours habituel des choses dans une culture donnée. Personne ne connaît exactement l'histoire de son évolution, comment ce don est né et a survécu. Nous savons seulement que c'est un outil irremplaçable qui donne sens à l'interaction humaine. (Bruner $2002: 77$ ) 
Dans cette interaction humaine, le fait que les récits de vie et de société s'ancrent dans un contexte d'auralité est une invitation pour chacun d'entre nous à « prêter l'oreille ». Tel était le mot d'ordre que Walter Benjamin reprenait lorsqu'il étudiait l'art du conteur Nicolas Leskov (Benjamin [1936] 2006 : 126). « Le conteur emprunte la matière de son récit à l'expérience : la sienne ou celle qui lui a été rapportée par autrui. Et ce qu'il raconte, à son tour, devient expérience en ceux qui écoutent son histoire ", écrit-il (Ibid. : 121). Son propos donne un aperçu de ce que nous appelons l'auralité. "Prêter l'oreille » est certainement une exigence dans la gestion politique, mais aussi pour les sciences sociales. L'ethnographie s'imagine mal sans une attention aux paroles échangées et entendues sur le terrain d'études. Le travail sur les contes mais aussi plus spécifiquement ethnographique d'Yvonne Verdier met particulièrement bien en relief l'importance de l'auralité24. De 1968 à 1975, elle mena avec son équipe une enquête en Côte d'Or dans le village de Minot. Elle y récolta la parole des femmes lorsque ces dernières réalisaient certaines tâches, par exemple tuer le cochon, ou d'autres plus régulières comme laver, coudre et cuisiner. Ces activités orientaient la discussion de ces femmes vers des sujets précis, des récits où leurs souvenirs refaisaient surface. Faire et dire étaient intimement liés, l'un déclenchant l'autre. Il semblait indispensable à Yvonne Verdier pour mener à bien son travail d'« entendre la langue » de ces femmes :

[...] dans le phrasé, un rythme souvent répétitif, non pas lent, car le plus souvent le mouvement en est alerte, une scansion propre aux gens de ce pays; dans le récit, où abondent les rappels, un génie de la mise en situation: toute affirmation est restituée comme dans un dialogue de théâtre. Il y a une manière de conter ou de parler à l'envolée, de crier contre les poules, les vaches ou les enfants, de converser au café ou de psalmodier le malheur vécu.

La parole dite et répétée se trouve être le fondement de la mémoire des choses comme de la mémorisation: «à Minot on sait "d'entendre dire", on connaît "de parole" ». (Verdier 1979: 11-12)

Ces formes littéraires ne renvoient jamais à un principe d'immanence qui ferait que le récit serait un objet clos.

Terminons par ces deux passages du livre d'Yvonne Verdier où, à la recherche d'analogies avec sa démarche, elle cite le peintre Millet qui dit la chose suivante à propos de sa toile Femme revenant du puits :

J'ai tâché de faire qu'on ne puisse la prendre ni pour une porteuse d'eau, ni pour une servante; qu'elle vienne puiser de l'eau pour l'usage de sa maison, l'eau pour en faire la soupe à son mari et à ses enfants ; qu'elle ait l'air de n'en porter ni plus ni moins lourd que le poids de ses seaux pleins [...] qu'elle accomplisse avec simplicité et bonhomie, sans le considérer comme une corvée, un acte qui est, avec les autres travaux du ménage, un travail de tous les jours et l'habitude de sa vie. Je voudrais bien qu'on imagine la fraîcheur du puits et que son air d'ancienneté fasse voir que beaucoup avant elles y sont venues puiser de l'eau. (Verdier 1979 : 14)

On saisit dans l'ambition de Millet la dimension narrative qu'il souhaite appliquer à son tableau et qui fait que sa toile devient une œuvre ouverte, « une mise en images de gestes » (Ibid.).

Dans la citation qui suit, Yvonne Verdier déploie le cheminement qui mène de la parole des femmes de Minot à une pensée où s'agitent des préoccupations que l'on oserait qualifier d'universelles :

Ce sont les femmes de Minot elles-mêmes qui [...] ont proposé la matière essentielle, elles aussi qui ont tendu le fil qui nous a guidée, ce qui nous a conduite c'est moins une méthode qu'une volonté de "les prendre au mot ": leur discours a ses raisons, mais ces raisons ne sauraient se dévoiler dans l'application d'une grille extérieure, 
et ce que l'on saisit à la volée, ou lors d'échanges et d'interrogations plus réfléchies, prend forme et révèle sa logique et sa cohérence dans une lente remontée mot à mot au cœur de ce qui est une pensée. S'y trouve agité ce qui nous agite tous, l'amour, la mort, le travail, le destin, la vie. En leur parole, elles possèdent et l'intelligence de leur propre réalité et le don de la transmettre. (Verdier 1979: 14-15) assaisonner fait toute la différence entre récit réussi et message de marketing.

\section{BIBLIOGRAPHIE}

Beck, Deborah, 2005, « Odysseus: Narrator, Storyteller, Poet? », Classical Philology, vol. 100, nº 3, p. 213-227.

Benjamin, Walter, 2006, Euvres, III, trad. M. de Gandillac, P. Rusch, R. Rochlitz, Paris, Gallimard, coll. « Folio/essai ».

Bergson, Henri, 1932, Les Deux Sources de la religion et de la morale, Paris, PUF.

Bruner, Jerome, 2000, Culture et modes de pensée. L'esprit humain dans ses œuvres, trad. Y. Bonin, Paris, Retz.

,- 2002, Pourquoi nous racontons-nous des histoires?, trad. J. Bonin, Paris, Retz.

Cassin, Barbara (dir.), 1986, Le Plaisir de parler. Étude de sophistique comparée, Paris, Minuit, coll. « Arguments ».

Cauquelin, Anne, 1999, L'Art du lieu commun. Du bon usage de la doxa, Paris, Seuil.

Citton, Yves, 2010, Mythocratie. Storytelling et imaginaire de gauche, Paris, Amsterdam.

Denning, Stephen, 2007, The Secret Language of Leadership: How Leaders inspire Action Through Narrative, San Francisco, Jossey-Bass.

Detienne, Marcel, 1981, L'Invention de la mythologie, Paris, Gallimard.

Flahault, François, 2001, La Pensée des contes, Paris, Anthropos.

Gangloff, Anne, 2008, « Les héros et les penseurs grecs des deux premiers siècles après J.-C. », dans Pallas. Revue d'Études Antiques, « Mythes et savoirs dans les textes grecs et latins ", $\mathrm{n}^{\circ} 78$, p. 153-168.

Goyet, Florence, 2006, Penser sans concept, fonction de l'épopée guerrière, "Iliade ", "Chanson de Roland », « Hôgen », et « Heiji monogatari », Paris, Champion.

Mackie, Hilary, 1997, « Song and storytelling: an Odyssean perspective », Transactions of the American Philological Association, $\mathrm{n}^{\circ}$ 127, p. 77-95.

Marcellini, Anne, 2010, «La chose la plus rapide sans jambes: Oscar Pistorius ou la mise en spectacle des frontières de l'humain ", Politix, $\mathrm{n}^{\circ}$ 23, p. 139-165.

Molino, Jean, 2003, Homo fabulator. Théorie et analyse du récit, Arles, Actes sud. 
Nagy, Gregory, 1994, Le Meilleur des Achéens. La fabrique du héros dans la poésie grecque archaïque, trad. J. Carlier et N. Loraux, Paris, Seuil.

Porcher, Louis et Groux, Dominique, 2013, Le Storytelling, un angle neuf pour aborder des disciplines multiples? Paris, L'Harmattan, coll. « Éducations comparées ».

Pucci, Pietro, 1995, Ulysse polutropos. Lectures intertextuelles de l'Iliade et de l'Odyssée, trad. J. RoutierPucci, Villeneuve d'Ascq, Presses Universitaires du Septentrion.

-, 1996, «The song of the Sirens », dans S. L. Schein (dir.), Reading the Odyssey, selected interpretative essays, Princeton, Princeton University Press, p. 191-200.

Rabatel, Alain, 2009, Homo narrans. Pour une analyse énonciative et relationnelle du récit, Limoges, Lambert-Lucas, coll. « Linguistiques ».

Russo, Joseph A. 1976, "Is Aural or Oral Composition the Cause of Homer's Formulaic Style? ", dans B. A. Stolz, R. S. Shannon et A. Arbor (dir.), Oral Literature and the Formula, Ann Arbor, University of Michigan Press, p. 31-54.

-, 1997, «A Jungian Analysis of Homer's Odyssey », dans P. Young Eisendrath et T. Dawsson (dir.), The Cambridge Companion to Jung, Cambridge, Cambridge University Press, p. 240-254.

Salmon, Christian, 2007, Storytelling. La machine à fabriquer des histoires et à formater les esprits, Paris, La Découverte.

Scodel, Ruth, 1998, « Bardic Performance and Oral Tradition in Homer », American Journal of Philology, vol. 119, $\mathrm{n}^{\circ}$ 2, p. 171-194.

Verdier, Yvonne, 1978, «Grands mères si vous saviez... : Le Petit chaperon rouge dans la tradition orale ", Cahiers de la littérature orale, $\mathrm{n}^{\circ} 4$.

-, 1979, Façons de faire, façons de dire. La laveuse, la couturière, la cuisinière, Paris, Gallimard.

\section{NOTES}

1. Formate-t-il les esprits? Nous pensons, avec Yves Citton (2010:68), que l'expression est un peu abusive.

2. Notre réserve concernant l'ouvrage de Salmon prolonge celle formulée par Yves Citton (2010: 68): «Malgré ses nombreux mérites, le livre de Christian Salmon repose largement sur la prémisse d'un péché originel marquant le fait même de raconter une histoire. »

3. Pour les expressions homo narrans et homo fabulator, voir Alain Rabatel (2009) et Jean Molino (2003).

4. La tradition des Vies d'Homère, si foisonnante dans l'Antiquité-les plus connues sont attribuées, certainement à tort, à Hérodote et Plutarque -, fût écartée dès que la philologie classique, celle de Wilamowitz, s'empara des textes homériques. Le texte ne pouvait s'éclairer que de lui même.

5. Présupposé dont on peut se demander s'il ne transparaît pas en filigrane dans l'étude de Salmon.

6. «La pression de l'instinct a fait surgir en effet, au sein même de l'intelligence, cette forme d'imagination qu'est la fonction fabulatrice ", écrit-il, p. 172.

7. Voir, La République, III, 411 a 6-8 et Les Lois, III, $680 \mathrm{~d} 3$.

8. VIII, 368 pour la terpsis produite par le chant de Démodokos, et XI, 334, XIII, 2 pour la klèsis du récit d'Ulysse.

9. Odyssée, I, 1. 
10. Voir Odyssée, IX-XI, 334.

11. Sur ces différents sens de poluainos, cf. XII, 184 ; XIV, 508 ; XXI, 110. Il existe un troisième sens de poluainos, « qui sait parler par codes ».

12. Pietro Pucci (1995: 320), traduit ainos par apologue.

13. XVII, 514, 518-521.

14. Voir Nagy (1994: 279-283).

15. On pourrait parler d'une manière kairique (de kairos, la saisie de l'occasion) de faire des récits.

16. Sur Ulysse en trickster, voir Russo (1997).

17. Sénèque, De Beneficiis, I, 2-3, trad. Joseph Baillard.

18. Pour une analyse plus approfondie de ce point, nous renvoyons à Anne Gangloff (2008: 157-158).

19. Voir "Quand Joe le plombier traîne ses casseroles ", Libération, 17 octobre 2008, http:// www.liberation.fr/planete/2008/10/17/quand-joe-le-plombier-traine-ses-casseroles_153758.

20. Ce dernier déclara lors d'une interview en 2012 à CBS : « C'était une erreur de croire que mon travail était seulement de mettre en place de bonnes politiques. C'est important, mais la nature de la fonction du président est aussi de raconter au peuple une histoire » (cité dans Porcher et Groux 2013 : 183).

21. Pour un aperçu de l'idéologie managériale appliquée au storytelling, on pourra se reporter aux différents ouvrages de Stephen Denning, notamment The Secret Language of Leadership: How Leaders inspire Action Through Narrative (2007), et à la théorie du leadership.

22. Ajoutons qu'il est maintenant correspondant de guerre au Moyen-Orient pour le site conservateur, pjtv.com.

23. On pensera au fameux carrosse/citrouille de Cendrillon.

24. Yvonne Verdier (1978) a donné une interprétation très stimulante du Petit chaperon rouge.

\section{RÉSUMÉS}

Si le storytelling, cette technique visant à marquer les esprits par la narration de récits se voulant édifiants et mobilisateurs est une arme du management, nous nous demandons s'il n'est pas possible de penser l'attachement proprement humain au fait de narrer et d'entendre des histoires dans une perspective plus englobante. Les récits, accordant une large place aux péripéties, peuvent se révéler plus subversifs que ne l'imaginent les contempteurs du storytelling. S'en priver, c'est, d'une certaine manière, accréditer une thèse très contestable selon laquelle la pensée est plus substantielle lorsqu'elle n'est pas narrative.

If storytelling, a technic using mobilizing and inspiring tales, is a management weapon, we will focus our topic on the possibility to think the proper human attachement for telling and hearing stories in a more inclusive perspective. Stories, giving emphasis to vicissitudes, may be more subversive than imagined by despisers of storytelling, mostly they are fundamental in an heuristic point of view for ethnographic research. Denying it, it is somehow endorsing a very questionable assumption that thought is more substancial when not narrative. 
INDEX

Mots-clés : storytelling, pensée narrative, Ulysse storyteller, auralité

Keywords : narrative thought, Odusseus storyteller, aurality

\section{AUTEUR}

FRANÇOIS DINGREMONT

École des hautes études en sciences sociales 